\title{
Model Checking GSM-Based Multi-Agent Systems
}

\author{
Pavel Gonzalez, Alessio Lomuscio \\ Department of Computing, Imperial College London \\ \{pavel.gonzalez09, a.lomuscio\}@imperial.ac.uk \\ Andreas Griesmayer \\ ARM, Cambridge \\ andreas.griesmayer@arm.com
}

\begin{abstract}
Artifact systems are a novel paradigm for implementing service oriented computing. Business artifacts include both data and process descriptions at interface level thereby providing more sophisticated and powerful service inter-operation capabilities. In this paper we put forward a technique for the practical verification of business artifacts in the context of multi-agent systems. We extend GSM, a modelling language for artifact systems, to multi-agent systems and map it into a variant of AC-MAS, a semantics for reasoning about artifact systems. We introduce a symbolic model checker for verifying GSM-based multi-agent systems. We evaluate the tool on a scenario from the service community.
\end{abstract}

\section{Introduction}

It has long been argued [20, 5] that agents are a fitting paradigm for web-services, and more generally service oriented computing (SOC). Indeed, agent-based research has contributed a wealth of techniques ranging from verification [16], protocols [21] and actual prototype implementations [1]. SOC is currently a fast moving research area with significant industrial involvement where highly scalable implementations play a key role. Agent-based solutions can shape developments in SOC if they remain anchored to emerging paradigms being put forward by the leading players in the area.

An emerging and increasingly popular paradigm being investigated in SOC is that of business artifacts [7]. In this approach data, not only processes, play a key part in the service description and implementations. While in traditional service composition, processes are advertised at interface level, in the artifact approach both processes and the data structures upon which processes are meant to operate are given equal prominence. Indeed, in e-commerce, e-business and e-government, documents and their structures play a fundamental role in characterising the state of the system as well as the various operations to be conducted on it by the agents. Abstracting entirely from the data as it is done in traditional business process modelling, it is argued, amounts to an excessive simplification that forces us to models that are no longer meaningful to be used with complex, information-oriented applications.

Guard-Stage-Milestone (GSM) has recently been put forward [14, 13] as a language for implementing business artifacts. In line with some of the AI and agent-based tradition, GSM is a fully declarative language. GSM provides a description of stages, which are clusters of topical activity pertaining to some artifact data-structure. Stages are governed by guards controlling their activation and milestones determining whether or not the stage goals have been reached. GSM models are interpreted by Barcelona [12], a platform that implements GSM's semantics, thereby generating the intended runs of the system under a given sequence of external events.

While business artifacts are an attractive methodology for developing business processes and GSMbased services are a rapidly evolving area of research, they currently lack fully-fledged automatic method-

To appear in EPTCS. 
ologies for verification, orchestration and choreography. In this paper we put forward and evaluate a technique and an prototype implementation for the practical verification of business artifacts from a multi-agent system perspective.

Specifically, we give a MAS-based formal model to GSM systems and define the model checking problem on this model. We observe the problem is undecidable in general, but note that as long as we can show the system operates within bounds, the problem is decidable. Within these parameters the methodology we report is sound and complete. We have built an implementation to verify automatically whether a GSM system, including a number of agents, satisfies given temporal-epistemic specifications which may include quantification over artifact instances. We test the technique against an application developed by IBM.

Several contributions have so far studied the verification problem from a theoretical perspective [8, 11, 2, 4]. The results obtained identify fragments of decidable settings either through restrictions on the specification language or the semantics. While these results are certainly valuable, they provide no constructive methodology for the practical verification of GSM-based systems.

Closer to the work here presented is [10] where we introduced GSMC, a model checker for GSM. However, the semantics of the underlying formalism is one of plain transition systems and no support for agents in the system is provided. With no agents being present, no support is offered for views and windows, two key concepts that we fully support here. Additionally, as our concern was focused purely on the artifact system, the specification language only supports temporal logic, thereby making impossible to verify the information-theoretic properties of agents throughout an exchange as we do here.

\section{The Guard-Stage-Milestone Artifact Model}

The Guard-Stage-Milestone (GSM) approach to artifact systems [7] is particularly suitable for large unstructured processes where users have the freedom to decide what actions they perform and in what order. GSM is substantially influencing the emerging Case Management Modelling Notation standard [17]. IBM Watson developed Barcelona, an application for modelling and execution of GSM-based artifact systems [12]. Barcelona provides a fully model-driven environment where a business operations model of an artifact system is created in a web-based design editor component, and then directly used for deployment on an execution engine.

We begin with a short intuitive description before we give formal definitions for a GSM model $\Gamma$ in line with [13]. Artifact systems form a conceptual basis for modelling and implementing business processes [7] and are given in terms of artifact types, which correspond to classes of key business entities. Each type has a lifecycle model, which describes the structure of the business process, and an information model, which gives an integrated a view of the business data and the progress of the business process. The artifact system interacts with its environment via events.

GSM provides a declarative, hierarchical mechanism for specifying lifecycle models. Figure 1 illustrates a typical lifecycle model. It is represented by the following core concepts: The boxes denote stages, which represent clusters of activity designed to achieve milestones (o), operational objectives that an artifact aims go achieve. A guard $(\diamond)$ triggers activities in a stage when a certain condition is fulfilled. Stages are organised hierarchically, where the roots are called top-level stages, the leaves are called atomic stages and the non-leaf nodes are called composite stages. Atomic stages can contain tasks that perform automated actions. Stages can run in parallel and own at least one milestone and one guard, while both milestones and guards belong to exactly one stage. A stage becomes open when one of its guards is fulfilled and closed when one of its milestones is achieved. A milestone can also be invalidated 


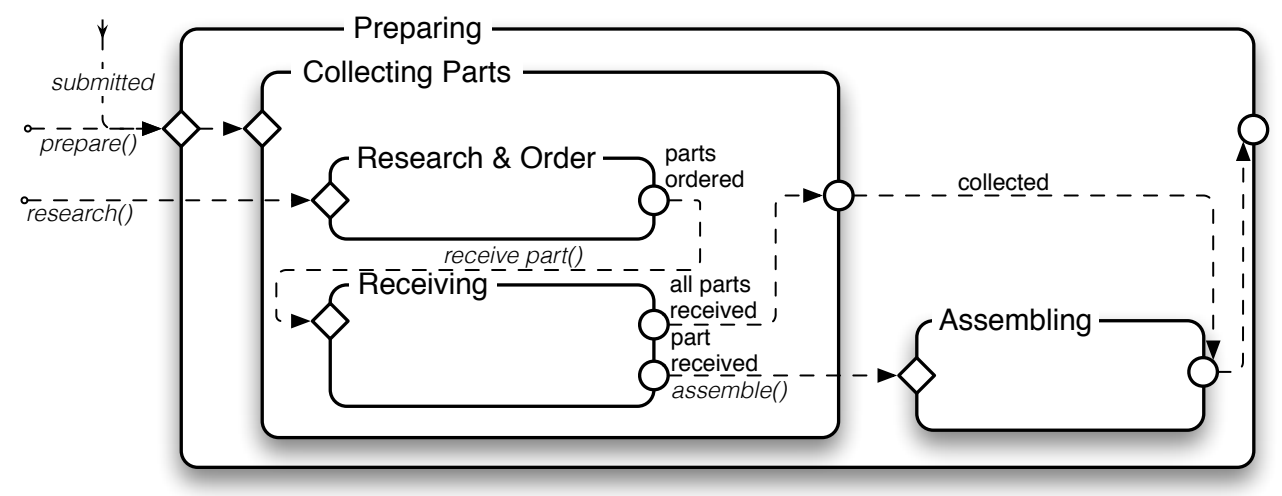

Figure 1: A lifecycle model.

when certain conditions are met.

The example in Figure 1 gives the portion of the lifecycle of a manufacturing process that handles the procuring of the required building parts and the organisation of the assembly. When a new order is received by the manufacturer, the submitted event is sent to the artifact system, which triggers the guard of the Preparing stage, and in turn starts with Collecting Parts. When this stage is open, an employee of the manufacturer researches the required components and sends the research event to the artifact system which in turn processes the order of the required parts. When a part is received (event part received), the Assembling of the available parts is triggered; when all parts are received and collected, the Preparing stage can be closed. More details on this lifecycle will be discussed in Section 6

In the following, we will define the artifact system more formally:

Definition 1 (Artifact Type) An artifact type $A T$ is a tuple $A T=\langle R, A t t, S t g, M s t, L c y c\rangle$ where

- $R$ is the name of the artifact type;

- Att is the information model as set of attributes;

- Stg is the set of stages;

- Mst is the set of milestones; and

- Lcyc is the lifecycle model.

The information model Att is partitioned into the set Att data of data attributes to hold business data and the set Att $t_{\text {status }}$ of status attributes to capture the state of the lifecycle model. Each stage (resp. milestone), has a Boolean status attribute in Att $t_{\text {status }}$, which is true iff the stage is active (resp. the milestone has been achieved). We write Dom for the domain of attributes in Att including the undefined value $\perp$. An artifact instance of an artifact type $A T$ is a tuple $\imath=\left(A T, A_{1}: c_{1}, \ldots, A_{k}: c_{k}\right)$, where $c_{i} \in \operatorname{Dom}\left(A_{i}\right)$.

The artifact system interacts with the environment by sending and receiving messages with payloads, where a message with a specific payload is called a typed external event.

Definition 2 (Event Type) An event type $E T$ is a tuple $E T=\left\langle E, A T, A_{1}, \ldots, A_{l}\right\rangle$, where

- $E$ is the name of the event type,

- AT is an artifact type, and 
- $A_{i} \in A t t_{\text {data }}$, where Att data is the set of data attributes of AT.

An event instance of an event type $E$ is a tuple $e=\left(l, A_{1}: c_{1}, \ldots, A_{l}: c_{l}\right)$, where $l$ is an artifact instance and $c_{i} \in \operatorname{Dom}\left(A_{i}\right)$ for $i \in[1 \ldots l]$. The tuple $p=\left(A_{1}: c_{1}, \ldots, A_{l}: c_{l}\right)$ is the payload of $e$. We now have all the ingredients to define a GSM model.

In addition to incoming external events, atomic stages have associated tasks that are invoked when a stage is opened and correspond to services that exist outside the artifact system. Agents are not directly present in the GSM model, but it is assumed that human or artificial entities perform tasks and generate events for the system. We will add a formalisation of agents in the next section.

Definition 3 (GSM Model) $A$ GSM model $\Gamma$ is a set of $n$ artifact types $A T_{i}$ for $i \leq n$ and $m$ event types $E T_{j}$ for $j \leq m$.

Definition 4 (Snapshot of GSM Model) $A$ pre-snapshot of $\Gamma$ is an assignment $\Sigma$ that maps each attribute $A \in A t t_{l}$ of each active artifact instance 1 to an element of $\operatorname{Dom}(A)$. A snapshot of $\Gamma$ is a presnapshot that satisfies the following GSM invariants: all sub-stages of a closed stage are closed; all milestones of an open stage are false; at most one milestone of a stage can be achieved at any time.

The operational semantics of GSM is based on the notion of a business step (B-step). This is an atomic unit that corresponds to the effect of processing one incoming event into the state of the artifact system. A B-step has the form of a tuple $\left(\Sigma, e, \Sigma^{\prime}, G e n\right)$, where $\Sigma, \Sigma^{\prime}$ are snapshots, $e$ is an incoming external event, and Gen is a set of outgoing external events generated by opening atomic stages during the B-step.

The progress of the lifecycle is driven by incoming events, which are called applicable if the lifecycle is ready to consume them. When an event is consumed by the artifact system, its payload is copied to the information model and the lifecycle model is updated. The opening of an atomic stage activates a task associated with the stage. It either performs an automated system task, such as the creation of a new instance, or corresponds to an operation outside the artifact system, in which case a service call is sent to the environment. In both cases, the task completion is marked by an event.

Both milestones and guards are controlled declaratively through sentries. A sentry of an artifact instance $\imath$ is an expression $\chi(l)$, which has one of the following three forms: "on $\xi(l)$ if $\varphi(l)$ "; "on $\xi(l)$ "; "if $\varphi(l)$ ", where $\xi(l)$ is an event expression for the triggering event $e$ of $l$ and $\varphi(l)$ is a wellformed formula over the instances occurring in the system. Milestones have one or more achieving sentries and optionally one or more invalidating sentries.

B-steps are computed according to the incremental semantics defined in [13] using a sequence of Prerequisite-Antecedent-Consequent (PAC) rules, which govern opening of stages and achieving of milestones. The PAC rules are formed from the sentries of the GSM model and restrictions are placed on the order of rule application, which in turns restricts the GSM model. A PAC rule for a GSM model $\Gamma$ is a tuple $\rho=(\pi, \alpha, \gamma)$, where: the prerequisite $\pi$ is a formula on the attributes in Att $t_{\text {status }}$; the antecedent $\alpha$ is a sentry based on attributes in Att, the internal events over Att status and external event types $E T$; the consequent $\gamma$ is an internal event $\odot \sigma$, where $\odot \in\{+,-\}$ is the update of a status attribute $\sigma \in$ Att $_{\text {status. }}$. In response to a single incoming external event, the artifact system fires all applicable rules until no rule can be fired.

To ensure the semantics is well-founded, an acyclicity condition is imposed on a GSM model $\Gamma$. This is defined by using the polarised dependency graph, denoted $P D G(\Gamma)$. A GSM model $\Gamma$ is well-formed if $P D G(\Gamma)$ is acyclic, i.e., there is no cyclic interdependence between the PAC rules of $\Gamma$. 


\section{Artifact-Centric Multi-Agent Systems}

To analyse interactions within a GSM-based artifact system, we use artifact-centric multi-agent systems (AC-MAS) [11, 3], a semantics based on interpreted systems [18, 9]. As GSM can in principle include multiple artifact instances, as it will be clear below, we require a limited form of quantification. We therefore introduce IQ-CTLK, an extended version of CTLK, which is frequently used to describe agents that share a common environment. IC-CTLK is a temporal-epistemic specification language with quantification over artifact instances.

\subsection{Formal Model}

In an AC-MAS a set of agents $\mathscr{A}$ share an environment $E$ constituted by the artifact system, i.e., the underlying elements of the environment are evolving artifacts of type $R$. The environment and an agent $i \in A$ have a local state ( $L_{E}$ and $L_{i}$ respectively), where the agent can observe parts of the environment (i.e., some of the artifact instances in it). The local state of an agent thus comprises private data for the agent and observable aspects of the artifact system. We write $l_{E}(s)$ to represent the local state of the environment in the global state $s$, and $l_{i}(s)$ to represent the local state of agent $i$.

Definition 5 (Environment) The environment represents an artifact system $A S$ and is a tuple $E=$ $\left\langle L_{E}\right.$, Act $\left._{E}, P_{E}\right\rangle$, where

- $L_{E}$ is the set of local states,

- Act $t_{E}$ is the set of local actions, which correspond to the interface of the AS, and

- $P_{E}: L_{E} \rightarrow 2^{A c t_{E}}$ is the environment's protocol function, which enables actions that can be performed depending on the local state of the AS.

An agent is defined formally as:

Definition 6 (Agent) An agent in an AS is a tuple $i=\left\langle L_{i}, A c t_{i}, P_{i}\right\rangle$, where

- $L_{i}$ is the set of local states including the observable aspect of the AS;

- $A c t_{i}$ is the set of local actions corresponding to events that can be sent by the agent onto the AS and including an action skip for performing a null action;

- $P_{i}: L_{i} \rightarrow 2^{\text {Act }}$ is the local protocol function.

An agent $i$ and the environment $E$ communicate by synchronisation on actions, where $A_{c t}$ corresponds to events enabled by the artifact system, and $A c t_{i} \subseteq A c t_{E} \cup\{$ skip $\}$ is the set of local actions corresponding to events that can be executed by the agent and the idle action skip. As in plain interpreted systems, protocols are used to select the actions performed in a given state, where $P_{E}: L_{E} \rightarrow 2^{\text {Act }_{E}}$ is the environment's protocol function, which enables executable events depending on the local state of the artifact system and $P_{i}: L_{i} \rightarrow 2^{\text {Act }_{i}}$ is the local protocol function of agent $i$.

Following the terminology of [3] we define an AC-MAS as the composition of the environment and a number of agents as follows:

Definition 7 (AC-MAS) Given an environment $E$ and a set of agents $\mathscr{A}$, an artifact-centric multi-agent system is a tuple $\mathscr{P}=\langle S, \mathscr{I}, \tau\rangle$, where

- $S \subseteq L_{E} \times L_{1} \times \cdots \times L_{n}$ is the set of reachable global states,

- I is the initial state, and 
- $\tau: S \times A c t \rightarrow 2^{S}$ with Act $=A c t_{E} \times A c t_{1} \times \cdots \times A c t_{n}$ is the global transition function. The transition $\tau(s, \alpha)$ is defined for $\alpha=\left(a_{E}, a_{1}, \ldots, a_{n}\right)$ iff $a_{E} \in P_{E}\left(l_{E}(s)\right)$, and $\exists_{0 \leq i<n}: a_{i} \in P_{i}\left(l_{i}(s)\right), a_{E}=$ $a_{i} \wedge \forall_{j \neq i}: a_{j}=$ skip.

Intuitively, the conditions on the transition relation limit the communication between agents and environment such that environment and agent agree on the same action. The environment enables actions when the artifact system is ready to consume them, while the agent $i$ decides on the actions to execute depending on a local strategy encoded in $P_{i}$. Only one agent can interact with the environment at a time while the others are idle.

We write $s \rightarrow s^{\prime}$ iff there exists an $\alpha$, such that $s^{\prime} \in \tau(s, \alpha)$, and call $s^{\prime}$ the successor of $s$. A run $r$ from $s$ is an infinite sequence $s^{0} \rightarrow s^{1} \rightarrow \ldots$ with $s^{0}=s$. We write $r[i]$ for the $i$-th state in the run and $r_{s}$ for the set of all runs starting from $s$. A state $s^{\prime}$ is reachable from $s$ if there is a run from $s$ that contains $s^{\prime}$. In line with the semantics of epistemic logic [9], we say that the states $s$ and $s^{\prime}$ are epistemically indistinguishable for agent $i$, or $\sim_{i}$, iff $l_{i}(s)=l_{i}\left(s^{\prime}\right)$.

\subsection{The Logic IQ-CTLK}

We are interested in specifying temporal-epistemic properties of agents interacting with the artifact system, as well as the system itself. Since GSM supports the dynamic creation of unnamed artifacts, the properties need to be independent of the actual number or possible IDs of artifact instances in the system. To specify such properties we here define a temporal-epistemic logic that supports quantification over the artifact instances. We call the logic IQ-CTLK, for Instance Quantified CTLK, where CTLK is the usual epistemic logic on branching time. It is a subset of FO-CTLK where quantification can only be over artifact instances but not data. The syntax is defined in BNF notation as follows:

$$
\begin{gathered}
\varphi::=p|\neg \varphi| \varphi \vee \varphi|E X \varphi| E G \varphi \mid E(\varphi U \varphi) \\
\left|K_{i} \varphi\right| \forall x: R \varphi \mid \exists x: R \varphi
\end{gathered}
$$

where $R$ is the name of an artifact type and $p$ is an atomic proposition over the agents' private data and the attributes of active instances that are specified in terms of instance variables bound by the quantification operators. The quantified instance variables range over the active instances of a given artifact type $R$ in the state where the quantification is evaluated and must be bound. We write $R(s)$ for the set of instances of type $R$ in $s$. The remaining CTL operators can be constructed by combination of the ones given above. For example, $A G \forall x: \operatorname{Order} A F K_{i} x$.sent encodes the property expressing that in any reachable state, agent $i$ will eventually know that the attribute sent is set to true for every active instance of type Order.

We inductively define the semantics of IQ-CTLK over an AC-MAS $\mathscr{P}$ as follows. A formula $\varphi$ is 
true in a state $s$ of $\mathscr{P}$, written $(\mathscr{P}, s) \models \varphi$, if:

$$
\begin{array}{ll}
(\mathscr{P}, s) \models p & \text { iff } p \in s \\
(\mathscr{P}, s) \models \neg \varphi & \text { iff }(\mathscr{P}, s) \models \varphi \\
(\mathscr{P}, s) \models \varphi_{1} \vee \varphi_{2} & \text { iff }(\mathscr{P}, s) \models \varphi_{1} \vee(\mathscr{P}, s) \models \varphi_{2} \\
(\mathscr{P}, s) \models E X \varphi & \text { iff } \exists_{s^{\prime}}: s \rightarrow s^{\prime} \wedge\left(\mathscr{P}, s^{\prime}\right) \models \varphi \\
(\mathscr{P}, s) \models E G \varphi & \text { iff } \exists_{r \in r_{s}}: \forall_{i \geq 0}:(\mathscr{P}, r[i]) \models \varphi \\
(\mathscr{P}, s) \models E(\varphi U \psi) & \text { iff } \exists_{r \in r_{s}}: \exists_{k \geq 0}:(\mathscr{P}, r[k]) \models \psi \wedge \\
(\mathscr{P}, s) \models K_{i} \varphi & \forall_{j<k}(\mathscr{P}, r(j)) \models \varphi \\
(\mathscr{P}, s) \models \forall x: R \varphi & \text { iff } \forall s^{\prime} \in S: s \sim{ }_{i}^{\prime} \Rightarrow\left(\mathscr{P}, s^{\prime}\right) \models \varphi \\
(\mathscr{P}, s) \models \exists x: R \varphi & \text { iff } \forall u \in R(s):(\mathscr{P}, s) \models \varphi[u / x] \\
& \text { iff } \exists u \in R(s):(\mathscr{P}, s) \models \varphi[u / x]
\end{array}
$$

Given an AC-MAS model $\mathscr{P}$ and an IQ-CTLK specification $\varphi$, the model checking problem concerns the decision as to whether the formula $\varphi$ holds at the initial state of $\mathscr{P}$, written $\mathscr{P}=\varphi$. In the context of our formal model, an AC-MAS $\mathscr{P}$ satisfies $\varphi$ if $(\mathscr{P}, \mathscr{I}) \models \varphi$. Intuitively this means that the model $\mathscr{P}$ satisfies $\varphi$ if $\varphi$ is true in the initial state of $\mathscr{P}$.

This was shown to be undecidable on similar semantic structures and more expressive logics [2]. In the following sections, we will achieve decidability by bounding the data and the number of instances present. We will also show the implementation of the technique to demonstrate its feasibility.

\section{Agent-Based GSM}

Naturally, a GSM program only deals with the machinery related to the artifact system but does not provide a description of the agents interacting with it. To conduct the verification of agent-based GSM systems via model checking, we define A-GSM as an extension of GSM with a set of external agents. The artifact system and agents communicate using events, where the available events for an agent depend on the current state. The system progresses by non-deterministically selecting an agent, which sends an event and triggers the execution of the AS. Selection of the event and execution of the AS are seen as one step, a stable state has no pending events. We give a formal mapping $f: A-G S M \rightarrow A C-M A S$, such that $f$ preserves satisfaction of formulas in the specification language IQ-CTLK.

\subsection{Agent Description}

Here we outline how the agents are specified and interact with GSM to define an A-GSM instance. The behaviour of an agent is determined by the permitted access to the artifact system AS and by local decisions regarding events to send. The former is determined by an agent's role, while the latter are defined for each agent individually.

The role is defined using the view $v$ for the visible attributes, the window $\omega$ to select the visible instances, and the set of events $\varepsilon$ that are accepted by AS. While $v$ and $\varepsilon$ are simple lists, $\omega_{i}(l)$ is a formula that is evaluated for a specific artifact instance $l$ and an agent $i$. The instance is exposed to the agent only if $\omega_{i}(l)$ evaluates to true. In addition to the role, the description of an agent also contains a 


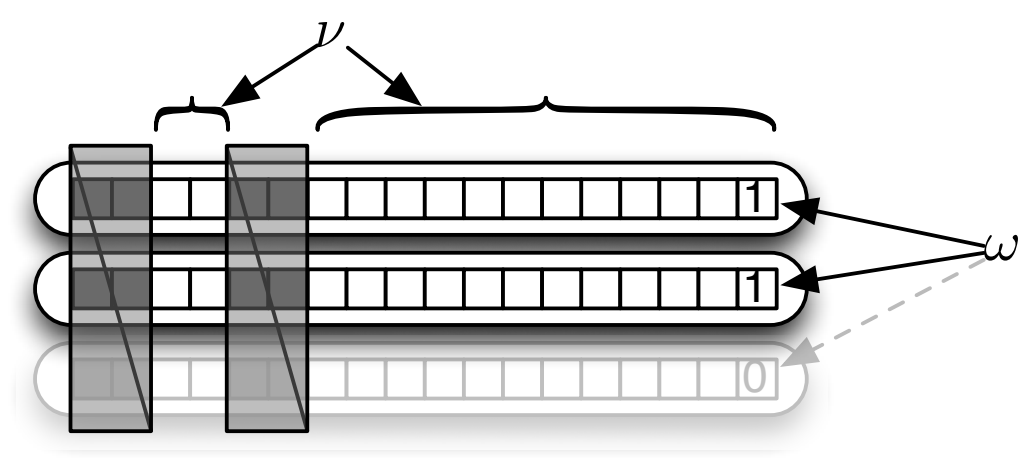

Figure 2: Static and Dynamic visibility in A-GSM.

protocol $\wp$ to determine its behaviour depending on the visible state of the AS, the agent's unique ID, and its private variables.

The concepts of $v, \omega$ and $\varepsilon$ are powerful tools to define the aspects agents can see and the ways they can interact with an artifact system. In Figure 2 the lines correspond to artifact instances that were created during run-time and the columns correspond to data attributes. $v$ defines a static view of the system, as it hides for each agent a fixed set of attributes depending on his role. For example, a Customer can only see that the state of an order moved from assembling to shipping, while a Manufacturer sees more detail, e.g., on suppliers. In contrast, $\omega$ gives a dynamic selection of the parts of the AS an agent can access in terms of the state of artifact instances as it hides complete instances depending on the current state. For instance, a Manufacturer may only see instances that represent unfinished orders while the window of a Customer can use the ID to restrict access to its own orders only.

Figure 3 outlines the description format. Visible data attributes are listed in the view field. The window field contains the formula for $\omega_{i}(\boldsymbol{l})$, where $\$ \$$ is a placeholder for the agent's ID. The field instantiation lists all artifact types that agents of this role may instantiate; the corresponding instantiation events are added to $\varepsilon$. To specify the status attributes and events that are added to $v$ and $\varepsilon$, the field transformation holds a set of GSM operators that allow to hide parts of the GSM model $\Gamma$. Valid commands are hide_stage_status ("S") and hide_milestone ("m") to hide the status attributes of stage $S$ and milestone $m$ respectively, and delegate_sentry ("s") to remove events from $\varepsilon_{i}$ if they are only used in sentry $s$. For convenience, the macro operators condense_stage("S") and eliminate_stage ("S") hide all sub-stages or all information including guards and milestones respectively.

The private variables of an agent are defined in a list var of variable names $\bar{x}$ with their type and initial value. The protocol lists entries of the form e : $\gamma \rightarrow \mu$ for all events $e$ the agent can send to the AS. If multiple entries for the same event are given, they are treated as disjunction. The condition $\gamma$ is given in terms of data attributes of the instance $l$, the payload, and the private variables. It defines the protocol function $\wp_{i}(l, \bar{x})$, which gives the set of events $e$ with their respective payloads that can be sent in the current state. The protocol also gives an update function $\mu_{i}(e, \bar{x})$, which computes new assignments for the local variables depending on the selected event and the local state of the agent. Note that by imposing conditions on the payload of an event $e, \wp$ also allows the agent to assign a specific value to its parameters, e.g., CustomerId is a parameter of Create_CO. 


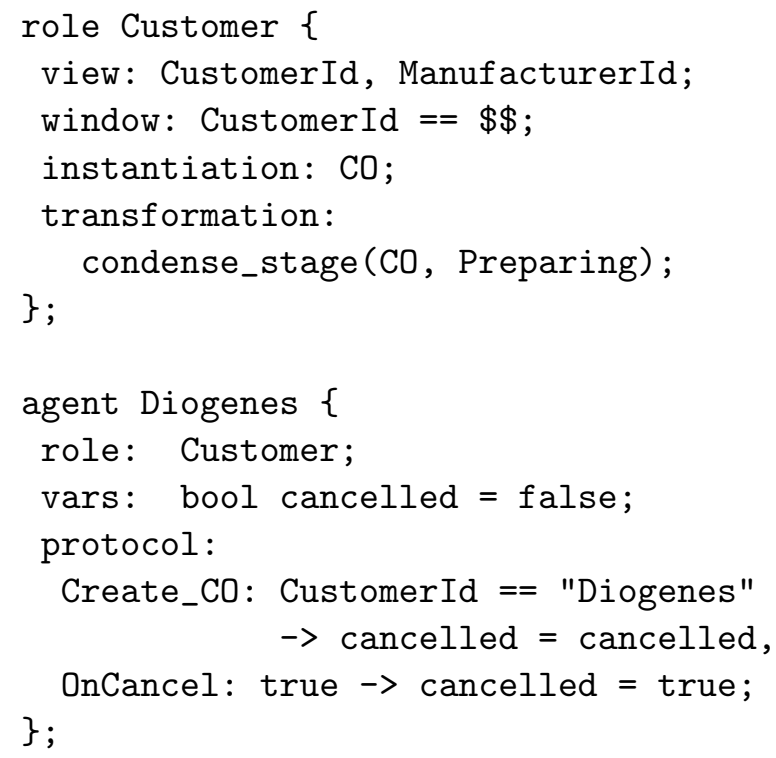

Figure 3: An agent definition file.

To handle automated tasks, we define an AutoAgent, which handles service calls and computations in the GSM model $\Gamma$ and returns the result to the artifact system in form of an event. The AutoAgent holds pending tasks in a buffer $t$, has full access to $\Gamma$, and can send the return messages at any time, but is otherwise handled like any other agent.

\subsection{Mapping to AC-MAS}

We now establish the formal mapping $f: A-G S M \rightarrow A C$-MAS. Note that the semantics for the local states and protocols of agents in A-GSM are given in terms of AC-MAS. We define the map by constructing the environment $\left\langle L_{E}, A c t_{E}, P_{E}\right\rangle$ from the GSM model $\Gamma$ of a given artifact system and creating an agent $\left\langle L_{0}, A c t_{0}, P_{0}\right\rangle$ for the AutoAgent, and $\left\langle L_{i}, A c t_{i}, P_{i}\right\rangle$ with $1 \leq i \leq n$ for each external A-GSM agent. We identify a GSM event $e$ with an AC-MAS action $a$ and will omit the conversion in the following for ease of presentation. The sets of actions $A c t_{E}, A c t_{0}$, and $A c t_{i}$ are thus directly defined by the events the AS provides and the permissions of the agents.

Global state: To construct a global AC-MAS state $\left(l_{E}, l_{0}, \ldots, l_{n}\right) \in S$ from an snapshot $\Sigma$, an AutoAgent buffer $t$ and the local agent states $x_{i}$, we identify $l_{E}$ with $\Sigma$ and $l_{0}$ with $t$. The local states $l_{1}, \ldots, l_{n}$ of the external agent comprise the state of the private variables $x_{i}$ and the projections $\Sigma_{\mid i}$ of the environment snapshot such that:

$$
\Sigma_{\mid i}=\left\{\imath \mid \exists_{\imath^{\prime} \in \Sigma}: \omega_{i}\left(\imath^{\prime}\right) \wedge \imath=\imath_{\mid v_{i}}^{\prime}\right\}
$$

where $\boldsymbol{l}_{\mid v_{i}}^{\prime}$ is the restriction of the artifact instance $\iota^{\prime}$ to the variables in $v_{i}$ (variables not in $v_{i}$ are replaced by $\perp$ ).

The initial state $\mathscr{I}$ is the empty state without any artifact instances in $\Sigma$ or pending tasks in $l_{0}$. Private variables are initialised to their initial value. 
Protocol: By construction, GSM executes only applicable events and blocks all others. Artifact instantiation events are always permitted. This is reflected in the environment protocol $P_{E}$ :

$$
P_{E}(\Sigma)=\left\{a \mid \exists_{\imath \in \Sigma}:(\chi \in X(\Gamma) \wedge \chi(l, a)) \vee a \in \text { inst }\right\}
$$

where $X(\Gamma)$ is the set of all sentries in the milestones and guards of $\Gamma$ and $\chi(\imath, a)$ is the evaluation of a sentry $\chi$ with respect to the action $a$ and status attributes $A t t_{\text {status }} \in \imath$. We write inst for the set of artifact instantiation events. The AutoAgent stores the set of pending tasks in its buffer $t$ and sends them at a later point to $\Gamma$. Thus, the protocol simply selects any pending task from its buffer by using the expression $P_{0}(t)=\{a \mid a \in t\}$. The protocol of an agent $i$ gives the set of actions that are available in visible instances of its local state and satisfy its local protocol:

$$
P_{i}\left(l_{i}\right)=\left\{a \mid \exists_{\imath \in l_{i}}: a \in \varepsilon_{i}(\imath) \cap \wp_{i}\left(\imath, x_{i}\right)\right\}
$$

These components suffice to instantiate a full AC-MAS from Definition 7. With these details in place we conclude the formal map from $A-G S M$ to $A C-M A S$. In the remainder of the paper we present an implementation of a model checker for $I Q-C T L K$ on $A C-M A S$.

\section{Implementation}

To perform AC-MAS model checking, we have implemented a model checking tool built on top of GSMC [10]. The checker, called Madrid ${ }^{1}$ is written in C++ and uses the CUDD library [22] for the back-end symbolic computations. Madrid builds the model and the transition relation and performs a symbolic state space exploration based on BDDs. The GSM model and the specification of the AutoAgent are directly loaded from the Barcelona XML input file; agent definitions are given in form of a configuration file as shown in Figure 3 .

To obtain finite state models, we introduce a bound on the number of instances that can be generated and use abstraction to create finite data. We allocate BDD variables for the states of the agents and the maximum number of artifact instances present in a run. In addition, we introduce an Overflow flag that indicates if the number of instances or data values were exceeded in a run. We pay special attention to this case because some of the results of the check may be unsound and require a re-check with higher bounds. We furthermore capture the Event ID and Payload of the next action $a$ that is to be executed. A special flag Created in each artifact instance indicates whether it was instantiated in the corresponding run.

Rather then exploring all possible runs of the system one by one, the tool uses a symbolic representation to directly operate on sets of states. Protocols, states, temporal and epistemic relations are thus encoded as Boolean formulas using BDDs (denoted as $\bar{\wp}, \bar{\omega}, \ldots$ ). Differently from the current state of the art [15], here we have to consider windows to correctly encode the epistemic relations. We define the Boolean formula $\bar{l}_{i}(\Sigma)$ to encode the set of local states $l$ of agent $i$ at GSM snapshot $\Sigma$ as follows:

$$
\bar{l}_{i}(\Sigma)=\bigvee_{l \in \Gamma}\left(\left(\exists_{x \notin v_{i}(l)}: \Sigma \wedge \bar{\omega}_{i}(l)\right) \vee \neg \bar{\omega}_{i}(l)\right)
$$

\footnotetext{
${ }^{1}$ The pre-compiled binaries of the tool can be downloaded from https://www.dropbox.com/s/tux0v10mg3c1nk4/ madrid.tar.gz
} 
For constructing the state space, the protocol is taken into account to ensure all transitions are the result of the execution of actions that are enabled at their respective states. We also ensure that no action operates on instances outside the agent's window. We encode the protocol for agent $i$ as:

$$
\bar{P}_{i}(\Sigma)=\bigvee_{\imath \in \Gamma}\left(\exists_{x \notin\left(v_{i} \cup \bar{a}\right)}: \bar{\wp}_{i}(\imath) \wedge \bar{l}_{i}(\Sigma) \wedge \bar{\omega}_{i}(l)\right)
$$

where $\bar{a}$ denotes the variables used for encoding the action.

Any IQ-CTLK formula $\varphi$ to be verified is first rewritten by replacing the quantification operators with formulas that range over the actual instances. However, because artifact instances are created dynamically at run-time, the number of active instances is not known a priori and needs to be considered in the formula. We use the expression created $(l)$ to check if an instance was created (the Created flag is set) and rewrite the quantified formulas as follows:

$$
\begin{aligned}
& \forall x: \varphi \Rightarrow \bigwedge_{\imath \in \Gamma}: \text { created }(\imath) \rightarrow \varphi \\
& \exists x: \varphi \Rightarrow \bigvee_{\imath \in \Gamma}: \text { created }(\imath) \wedge \varphi
\end{aligned}
$$

Note that, for any existential formula to be valid, at least one of the artifact instances needs to be active; this is not the case in the initial state because no artifact instance has been created yet. Quantifiers can be arbitrarily nested and are resolved recursively. Once the details above are considered, Madrid follows existing methodologies to perform the verification of temporal-epistemic formulas [15].

\subsection{Limitations}

Note that the bound in the number of instances restricts the possible behaviour of the system, while data abstraction leads to an over approximation. This may lead to loss of soundness or completeness when the limits of the artifact instances are reached. The exact outcome depends on the type of the property considered. A violation of a universal property, for instance, does denote a violation on the full unbounded model even if the bound was exceeded during the computation. To the contrary, if an existential property is not satisfied, no conclusion can be drawn regarding the full model in general. These are limitations in the technique at present but, as we show in the next section, interesting scenarios can still be analysed.

\section{Experimental Results}

We evaluated Madrid on the Order-to-Cash scenario, a simplified version of the IBM back-end order management application supplied by IBM Research [13]. In this scenario a manufacturer schedules the assembly of a product based on a confirmed purchase order from a customer. Typically, a product requires several components that are sourced from different suppliers. After all components have been delivered the product is assembled and shipped to the customer.

The GSM program is specified in the form of a single-artifact Barcelona schema consisting of 9 stages and 11 milestones. To verify the model we performed small modifications to abstract from concrete products and created three agent roles for the above scenario: 1) a Customer who creates an artifact instance that represents the order and can only see instances they created;2) a Manufacturer who fulfils the order and can see only uncompleted instances of orders sent to him by a customer; and 3) a 
Table 1: Properties of the Order-To-Cash case study.

$$
\begin{aligned}
& A G \forall x: C O((x . B I d=\text { Dio } \wedge \neg x . \text { Cancelled }) \\
&\left.\rightarrow K_{\text {Dio }} E F \text { x.Received }\right) \\
& E F \quad \exists x: C O\left(x . B I d \neq \text { Dio } \wedge K_{\text {Dio }} x . \text { Received }\right) \\
& A G \forall x: C O((x . B I d=\text { Dio } \wedge x . \text { Ready }) \\
& \quad \rightarrow K_{\text {Dio } x . P a r t s=3)} \\
& E F \quad \exists x: C O(x . \text { BId }=\text { Dio } \wedge x . \text { Cancelled } \\
&\quad \wedge \neg \text { Dio.cancelled })
\end{aligned}
$$

Carrier who ships the finished product to the customer, and who can see only instances of orders that are to be shipped via them.

Figure 1 gives the lifecycle of the Preparing stage. It is controlled solely by the manufacturer, who, upon receiving the order, launches a research process to identify suitable suppliers and orders the required components. The assembling process can begin when the first component is received and remains active until all the components are collected. This is modelled by introducing a counter; the process is considered complete when 3 components have arrived.

Table 1 gives the properties we checked for different numbers of agents and artifact instances, where Dio is a customer agent (Diogenes) and $C O$ stands for the CustomerOrder artifact type. Property (1) represents that Diogenes knows that the product can always be received in all of his orders as long as they are not cancelled (i.e., that there is no deadlock in processing the order). To check that the order is private to the customer, property (2) expresses that Diogenes may know a product is received for an order with different owner. Property (3) encodes the ability of an agent to deduce information it can not directly observe by checking if Diogenes always knows there are 3 Parts collected in all of his orders when the milestone Ready is achieved. Property (4) implies that an agent other than Diogenes can cancel an order that belongs to Diogenes. This is done by using a private variable, which is set true only if Diogenes executed the Cancelled event.

We ran the tests on a 64-bit Fedora 17 Linux machine with a $2.10 \mathrm{GHz}$ Intel Core i7 processor and 4GB RAM and measured the number of reachable states, memory used, and CPU time required. The model checker evaluated the properties (1) and (3) to be true and the properties (2) and (4) to be false in the model. This is in line with our intuition of the model and shows that the GSM program of Order-toCash application is indeed correct with respect to the requirements.

Table 2 reports the performance for 3 agents (one for each role) and 15 agents respectively (6 customers, 5 manufacturers, and 4 carriers). We see that the run-time grows exponentially with the number of artifact instances, while the number of agents influences the resource usage only moderately. This is because additional agents add fewer states than additional artifact instances. The results show that the tool has the ability to effectively handle large state spaces, which is required to model realistic artifact systems with complex agent interactions. 
Table 2: Reachable states, memory and time usage for different numbers of artifact instances $\imath$ and agents.

\begin{tabular}{|c|c|c|c|c|c|c|}
\hline \multirow[b]{2}{*}{$\# \imath$} & \multicolumn{3}{|c|}{3 agents } & \multicolumn{3}{|c|}{15 agents } \\
\hline & \#states & $\mathrm{MB}$ & s & \#states & MB & $\mathrm{s}$ \\
\hline 1 & $1.17 \mathrm{e} 2$ & 27 & 0.1 & 2.92 e 3 & 31 & 0.2 \\
\hline 2 & $3.71 \mathrm{e} 3$ & 52 & 0.7 & 4.16 e 6 & 70 & 4.9 \\
\hline 3 & $1.16 \mathrm{e} 5$ & 64 & 5.9 & 5.82 e 9 & 84 & 65.5 \\
\hline 4 & $3.67 \mathrm{e} 6$ & 96 & 42.1 & $8.01 \mathrm{e} 12$ & 222 & 360.2 \\
\hline 5 & $1.18 \mathrm{e} 8$ & 195 & 176.7 & $1.09 \mathrm{e} 16$ & 539 & 1419.6 \\
\hline
\end{tabular}

\section{Conclusions}

In this paper we put forward a technique for the practical verification of GSM-based MAS. The approach consists of defining a formal map from the declarative, executable language GSM to an extension of previously studied artifact-centric MAS, a semantics for reasoning about MAS in a quantified setting of the artifact system environment. We reported on a fully-fledged model checker that implements this formal map and supports temporal-epistemic specifications in which quantification is allowed over artifact instances. The experimental results obtained against the Order-to-Cash application led us to conclude that the practical verification of reasonably sophisticated GSM-based MAS is feasible and scalable in valuable scenarios in business processes and services. However, GSM and Barcelona are still a topic of active research and development and sophisticated and stable models are hard to come by. A current line of work is therefore the evaluation of the approach on case studies of the EU FP7 projects ACSI (FP7-ICT-257593), which also supported the research on the tool.

We plan to extend the work reported here in a number of ways, including the support of limited forms of quantification over the data. Theoretical studies [11, 3] point to high-undecidability in settings where unbounded data is present. For this reason we will work on existential abstraction and data abstraction to achieve a transfer of the verification outcome from abstract to concrete models. In particular we work on 3 valued abstraction [19], an abstraction technique that supports the detection of insufficient information in the abstraction. At a later stage we wish to combine these with counter-example guided refinement procedures [6].

\section{Acknowledgements}

This research was supported by the EU FP7 projects ACSI (FP7-ICT-257593). Work by the author Andreas Griesmayer was conducted in part at Imperial College London and supported by the Marie Curie Fellowship "DiVerMAS” (FP7-PEOPLE-252184). Alessio Lomuscio acknowledges support from the UK Engineering and Physical Sciences Research Council through the Leadership Fellowship grant "Trusted Autonomous Systems" (EP/I00520X/1). 


\section{References}

[1] M. Baldoni, C. Baroglio \& V. Mascardi (2010): Special Issue: Agents, Web Services and Ontologies: Integrated Methodologies. Journal of Multiagent and Grid Systems 6(2), pp. 103-104. Available at http://dx.doi.org/10.3233/MGS-2010-0143

[2] F. Belardinelli, A. Lomuscio \& F. Patrizi (2011): Verification of Deployed Artifact Systems via Data Abstraction. In: Proceedings of International Conference on Service oriented Computing (ICSOC'11), LNCS 7084, pp. 142-156. Available at http://dx.doi.org/10.1007/978-3-642-25535-9_10

[3] F. Belardinelli, A. Lomuscio \& F. Patrizi (2012): An Abstraction Technique for the Verification of ArtifactCentric Systems. In: Proceedings of Principles of Knowledge Representation and Reasoning (KR'12), pp. 319-328.

[4] F. Belardinelli, A. Lomuscio \& F. Patrizi (2012): Verification of GSM-based artifact-centric systems through finite abstraction. In: Proceedings of International Conference on Service oriented Computing (ICSOC'12), LNCS 7636, pp. 17-31.

[5] T. Bultan, J. Su \& X. Fu (2006): Analyzing Conversations of Web Services. IEEE Internet Computing 10(1), pp. 18-25. Available at http://doi.ieeecomputersociety.org/10.1109/MIC.2006.1

[6] E. M. Clarke, O. Grumberg, S. Jha, Y. Lu \& H. Veith (2003): Counterexample-guided abstraction refinement for symbolic model checking. Journal of the ACM 50(5), pp. 752-794. Available at http://doi.acm.org/ 10.1145/876638.876643.

[7] D. Cohn \& R. Hull (2009): Business Artifacts: A Data-Centric Approach to Modeling Business Operations and Processes. Bulletin of the IEEE Computer Society Technical Committee on Data Engineering 32(3), pp. 3-9.

[8] A. Deutsch, L. Sui \& V. Vianu (2007): Specification and Verification of Data-Driven Web Applications. Journal of Computer and System Sciences 73(3), pp. 442-474.

[9] R. Fagin, J. Y. Halpern, Y. Moses \& M. Y. Vardi (1995): Reasoning About Knowledge. The MIT Press.

[10] P. Gonzalez, A. Griesmayer \& A. Lomuscio (2012): Verifying GSM-based Business Artifacts. In: Proceedings of the IEEE International Conference on Web Services (ICWS'12), pp. 25-32.

[11] B. Bagheri Hariri, D. Calvanese, G. De Giacomo, A. Deutsch \& M. Montali (2012): Verification of Relational Data-Centric Dynamic Systems with External Services. Technical Report, CoRR Technical Report, abs/1203.0024, arXiv.org.

[12] F. T. Heath, R. Hull \& R. Vaculín (2011): Barcelona: A design and runtime environment for modeling and execution of artifact-centric business processes (demo). In: Proceedings of the International Conference on Business Process Management (BPM'11).

[13] R. Hull, E. Damaggio, R. De Masellis, F. Fournier, M. Gupta, F. T. Heath, S. Hobson, M. H. Linehan, S. Maradugu, A. Nigam, P. N. Sukaviriya \& R. Vaculín (2011): Business artifacts with guard-stage-milestone lifecycles: managing artifact interactions with conditions and events. In: Proceedings of the International Conference on Distributed Event-Based Systems (DEBS'11), pp. 51-62.

[14] R. Hull, E. Damaggio, F. Fournier, M. Gupta, F. T. Heath, S. Hobson, M. H. Linehan, S. Maradugu, A. Nigam, P. N. Sukaviriya \& R. Vaculin (2011): Introducing the Guard-Stage-Milestone Approach for Specifying Business Entity Lifecycles. In: Proceedings of the Internationsn Workshop on Web Services and Formal Methods (WS-FM'10), LNCS 6551.

[15] A. Lomuscio, H. Qu \& F. Raimondi (2009): MCMAS: A Model Checker for the Verification of Multi-Agent Systems. In: Proceedings of Computer Aided Verification (CAV'09), LNCS 5643.

[16] A. Lomuscio, H. Qu \& M. Solanki (2012): Towards Verifying Contract Regulated Service Composition. Journal of Autonomous Agents and Multi-Agent Systems 24(3), pp. 345-373.

[17] Object Management Group (2012): Proposal for: Case Management Modeling and Notation (CMMN) Specification 1.0. Document bmi/12-02-09. 
[18] R. Parikh \& R. Ramanujam (1985): Distributed Processes and the Logic of Knowledge. In: Logic of Programs, LNCS 193, pp. 256-268.

[19] Sharon Shoham \& Orna Grumberg (2008): 3-Valued abstraction: More precision at less cost. Information and Computation 206(11), pp. 1313 - 1333, doi:10.1016/j.ic.2008.07.004. Available at http: //www.sciencedirect.com/science/article/pii/S089054010800093X

[20] M. Singh, A. S. Rao \& M. Georgeff (1999): Formal Methods in DAI: Logic-Based Representation and Reasoning. In Gerhard Weiß, editor: Multiagent Systems: A Modern Approach to Distributed Artifical Intelligence, MIT Press, pp. 331-376.

[21] M. P. Singh \& M. N. Huhns (2005): Service-oriented computing - semantics, processes, agents. Wiley.

[22] F. Somenzi (2012): CUDD: CU Decision Diagram Package - Release 2.5.0. http://vlsi.colorado. edu/ fabio/CUDD/. January 2013. 